\title{
Experimental evaluation of spatial capture-recapture study design
}

\author{
Jill Fleming (iD) ${ }^{1,2,5}$ Evan H. Campbell Grant iD,${ }^{1}$ Sean C. Sterrett (D) ${ }^{3}$ and Chris Sutherland (D) 2,4 \\ ${ }^{1}$ USGS Eastern Ecological Science Center, SO Conte Anadromous Fish Laboratory, 1 Migratory Way, Turners Falls, Massachusetts \\ 01376 USA \\ ${ }^{2}$ Department of Environmental Conservation, University of Massachusetts, 160 Holdsworth Way, Amherst, Massachusetts 01003 USA \\ ${ }^{3}$ Department of Biology, Monmouth University, 400 Cedar Avenue, West Long Branch, New Jersey 07764 USA \\ ${ }^{4}$ Centre for Research into Ecological \& Environmental Modelling, The Observatory, Buchanan Gardens, University of St Andrews, \\ St Andrews, Fife KY16 9LZ United Kingdom
}

Citation: Fleming, J., E. H. C. Grant, S. C. Sterrett, and C. Sutherland. 2021. Experimental evaluation of spatial capture-recapture study design. Ecological Applications 00(00):e02419. 10.1002/eap.2419

\begin{abstract}
A principal challenge impeding strong inference in analyses of wild populations is the lack of robust and long-term data sets. Recent advancements in analytical tools used in wildlife science may increase our ability to integrate smaller data sets and enhance the statistical power of population estimates. One such advancement, the development of spatial capture-recapture (SCR) methods, explicitly accounts for differences in spatial study designs, making it possible to equate multiple study designs in one analysis. SCR has been shown to be robust to variation in design as long as minimal sampling guidance is adhered to. However, these expectations are based on simulation and have yet to be evaluated in wild populations. Here we conduct a rigorously designed field experiment by manipulating the arrangement of artificial cover objects (ACOs) used to collect data on red-backed salamanders (Plethodon cinereus) to empirically evaluate the effects of design configuration on inference made using SCR. Our results suggest that, using SCR, estimates of space use and detectability are sensitive to study design configuration, namely the spacing and extent of the array, and that caution is warranted when assigning biological interpretation to these parameters. However, estimates of population density remain robust to design except when the configuration of detectors grossly violates existing recommendations.
\end{abstract}

Key words: red-backed salamanders; spatial capture-recapture; standardization; study design.

\section{INTRODUCTION}

For many species, the need for informed and effective conservation management is urgent, and the establishment of long-term standardized monitoring in the present does not advance the identification of beneficial management actions. Oftentimes, management decisions need to be made quickly, and may only be informed by data that are readily available (Lyons et al. 2010). Where existing data are derived from different study designs and violate standardization assumptions, analyzing those data in a statistical framework is complicated, and without accounting for design differences explicitly, researchers risk mischaracterizing true drivers of variation among studied populations due to biases imposed by source study designs. In response to disparities in sampling methods of existing data sets, there have been repeated calls for development of rigorous analytical methods that are "data inclusive" and able to estimate key population parameters with data derived from inconsistent sampling designs (Beebee and Griffiths 2005). Understanding the influence of study design on

Manuscript received 31 January 2020; revised 13 January 2021; accepted 3 March 2021; final version received 30 June 2021. Corresponding Editor: Beth Gardner.

${ }^{5}$ E-mail: jefleming@usgs.gov statistical inference is itself an important endeavor, but is a particularly important consideration when integrating data across monitoring efforts, a process that empowers researchers to make the broad and multi-scale inference essential to both local and range-wide management of species in or at risk of decline.

Capture mark-recapture (CMR) techniques have revolutionized the study of demographic processes and the ability to understand the dynamics of wild populations (Williams et al. 2002). However, a key assumption of traditional CMR is that detection probability is homogeneous throughout the trapping area, which may be violated if individuals are exposed more frequently, e.g., because of where they live, to trapping (Efford 2004). Further, these traditional methods ignore inherent spatial information built into many CMR sampling designs.

Spatial capture-recapture (SCR) methods (Efford 2004, Royle and Young 2008) extend classical CMR models to exploit spatially explicit individual encounter histories (individual-by-trap-by-occasion detections) and improve estimates of population density. Fundamental to this development is that SCR explicitly integrates the spatial sampling design of a study and the spatial ecology of the focal species, offering a statistical framework for investigating a wide range of spatially structured ecological processes (Royle et al. 2018), 
including absolute population density without the need for arbitrary ad hoc adjustments such as the maximum mean distance moved (MMDM or derivations of this method such as one-half MMDM; Otis et al. 1978). SCR offers an inferential framework that naturally facilitates comparisons of estimated state variables obtained from across a species' geographic range (Borchers and Efford 2008). This is a critical advancement because, to date, range-wide monitoring of populations and evaluations of conservation status often lack the standardization necessary to produce consistent and comparable data across space and time.

Given the explicit definition of space in the SCR model, spatial sampling design may be particularly consequential, especially as it relates to the number and spacing of traps. Precision of density estimates is optimized by maximizing the number of individuals detected, which is informative about density, and the number of spatial recaptures (i.e., captures of individuals at $>1$ location), which are informative about the spatial heterogeneity in detectability (Sollmann et al. 2012). The number of traps may determine the number of individuals encountered and the frequency of detections, whereas the trap spacing may determine the frequency and spatial range of spatial recaptures. In the sampling design phase, the choice between deploying an extensive trapping array (i.e., large area, low trap density) versus an intensive array (i.e., small area, high trap density) may have substantial implications for detection probability and number of spatial recaptures (Wilton et al. 2014, Dupont et al. 2021). While empirical evaluations of SCR design are lacking, simulation studies suggest that SCR methods perform well when the trapping area is larger than a home range, and when trap spacing is close to $2-3 \sigma$, which, in theory, equates roughly to the radius of a typical home range, making spatial recaptures possible (Sollmann et al. 2012, Sun et al. 2014). Simulations also suggest that SCR is robust to differences in sampling design when these rules of thumb are adhered to, although this has yet to be evaluated empirically in wild populations, likely due to the logistical challenge of experimentally manipulating design configurations. While simulations are informative of model performance, empirical evaluations of study designs are critical for revealing unknown sources of variation such as behavioral interactions. If important sources of variation are not known, and therefore not accounted for, results of simulations may be misleading.

Here we conduct a rigorously designed field experiment in which we manipulate the arrangement of traps used to collect spatially explicit capture data and empirically evaluate the effects of design configuration on inference made using SCR. In our experiment, we expose three independent wild populations of redbacked salamanders (Plethodon cinereus) to sampling via five unique spatial configurations of traps and compare array-specific estimates of SCR model parameters to evaluate the effect of study design.

\section{Materials AND Methods}

\section{Model system}

While the red-backed salamander is considered common throughout its range, abundance may be highly variable (see Peterman and Semlitsch 2013, HernándezPacheco et al. 2019), necessitating unbiased methods for comparing independent populations. The species has been studied extensively throughout its range, but rangewide comparisons of wild population parameters has been limited by lack of standardization. The traps used in our experiment are a common terrestrial salamander sampling method, artificial cover objects (ACOs; also known as coverboards), which are materials (e.g., wood, tin, carpet) placed on a soil surface to mimic natural refugia such as rocks and woody debris, providing predictable opportunities for capture (Hesed 2012). ACOs are typically arranged in grids and have a demonstrated ability to produce spatially explicit density estimates (Muñoz et al. 2016b, Sutherland et al. 2016). For most animals, the issue of scale is problematic because movement (i.e., the spatial scale of detection) is logistically intensive to observe in large home ranges. Compared to other organisms studied with mark-recapture techniques, red-backed salamanders have exceptionally small home ranges that require little effort to observe in entirety (3-28 m²; Muñoz et al. 2016, Sutherland et al. 2016). The red-backed salamander's small home ranges and the ability of ACOs to be easily manipulated provides an ideal system for empirically evaluating the sensitivity of SCR-derived estimates of density under different trap configurations. While the absolute spatial scale of the red-backed salamander system is relatively small, the number of traps, the population density, and spatial extent relative to home range size, are representative of typical SCR studies, and therefore insights from this experiment should be general and of broad interest.

\section{Study area}

This study took place in Wendell State Forest (Wendell, Massachusetts, USA), which is managed by the Massachusetts Department of Conservation and Recreation (DCR). Wendell State Forest is in northcentral Massachusetts and spans 7,566 acres. Previous surveys performed here confirmed the presence of redbacked salamanders (USGS ARMI, unpublished data), and three experimental study sites were established within the forest (Fig. 1).

Stand composition of the three study sites were similar and included mixed hardwoods (Quercus alba, Q. rubra, Q. veluntina, Betula alleghaniensis, B. lenta) with hemlock (Tsuga canadensis) and pines (Pinus resinosa, P. strobus), a dominant forest type in the red-backed salamander's range (Nichols 1935). Study areas were cleared of downed logs to standardize the distribution of refugia. 


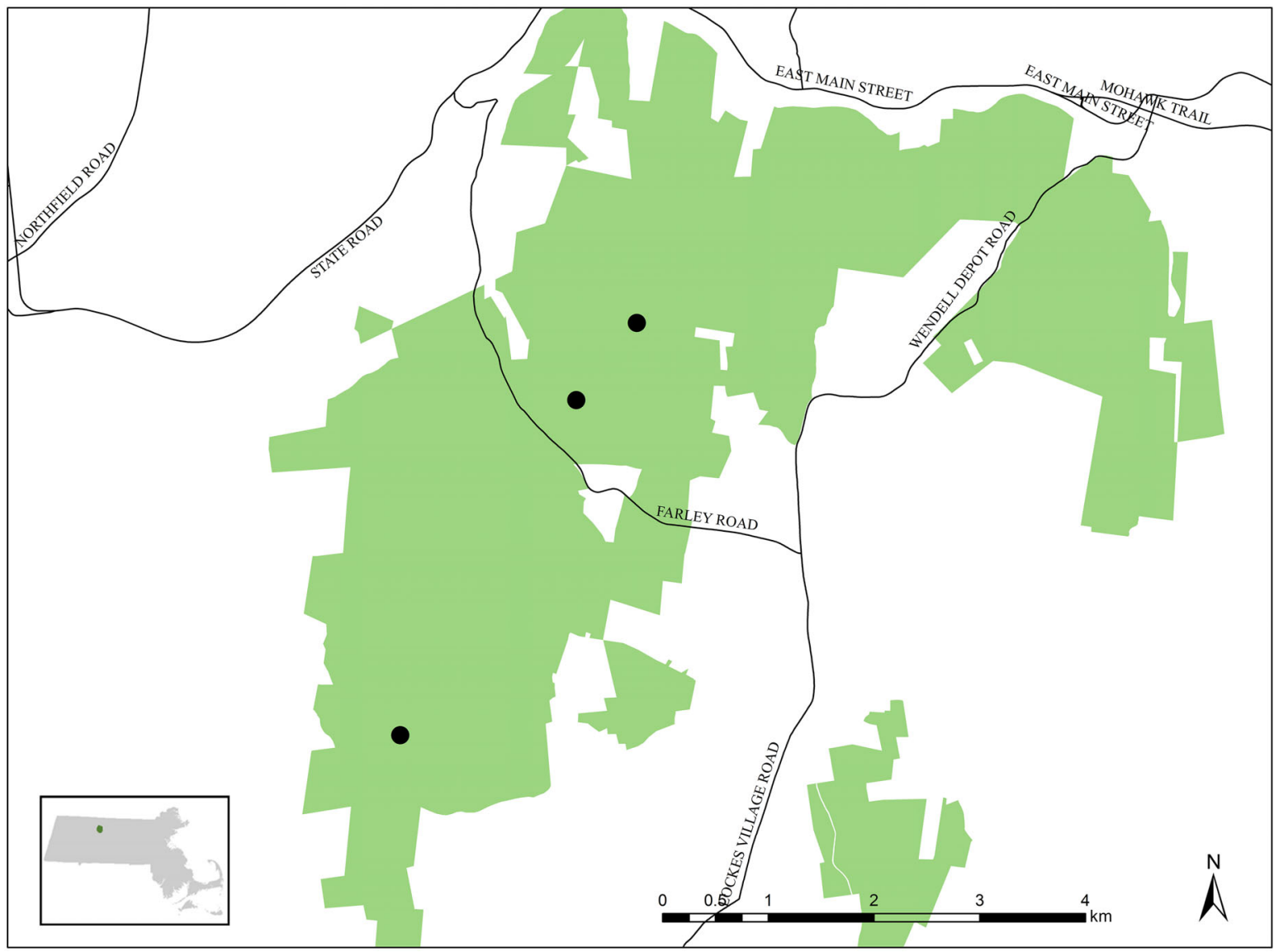

FIg. 1. Three sites within Wendell State Forest in which five experimental configurations of artificial cover object (ACO) arrays were installed.

\section{Experimental design}

Sites were selected to represent three independent populations and were spaced $\geq 1 \mathrm{~km}$ apart (Fig. 1; Cabe et al. 2007). The ACOs used in this experiment were wood squares $(30 \times 30 \times 2.5 \mathrm{~cm})$ and were arranged in gridded arrays. Within each site, five ACO arrays (15 arrays total) were established close enough to assume limited variation in density (i.e., sampling of a single population), but far enough ( $\geq 25 \mathrm{~m}$ ) to avoid capture of individuals in multiple arrays within a sampling season (i.e., maintain independence). As a control, we use an ACO array with 1-m spacing, the official protocol used by SPARCnet (Salamander Population and Adaptation Research Collaboration network), a network of many researchers and educators, and 139 ACO arrays distributed throughout the red-backed salamander range. The four experimental ACO designs were chosen to reflect deviations of the home range coverage and trap spacing recommendations. Specifically, we manipulated the total area (size) of the ACO array to evaluate the effects of variation in the spatial coverage with respect to red-backed salamander home range (array: control-size, large, and small), and ACO spacing to evaluate the effects of variation in trap spacing with respect to the scale of space use (control, sparse, and dense). Recent SCR-derived estimates of red-backed salamander $95 \%$ home range sizes range from 3 to $28 \mathrm{~m}^{2}$ based on corresponding estimates of the space use parameter, $\sigma$, of 1-3 m, respectively (Muñoz et al. 2016b, Sutherland et al. 2016). The five experimental ACO array designs were (Fig. 2)

1) a control array as a rectangle with ACOs spaced $1 \mathrm{~m}$ apart $(7 \times 5 \mathrm{~m}$ with $35 \mathrm{ACOs})$;

2) a control-sized sparse array with the same area as the control, but with ACOs spaced $2 \mathrm{~m}$ apart $(7 \times 5 \mathrm{~m}$ with 12 ACOs);

3) a large sparse array has the same number of ACOs as the control, but with ACOs spaced $2 \mathrm{~m}$ apart $(10 \times 14 \mathrm{~m}$ with $35 \mathrm{ACOs})$;

4) a control-sized dense array is the same area as the control with ACOs spaced $0.5 \mathrm{~m}$ apart $(7 \times 5 \mathrm{~m}$ with 117 ACOs);

5) a small dense array has the same number of ACOs as the control, but is spaced $0.5 \mathrm{~m}$ apart $(2.5 \times 3.5 \mathrm{~m}$ with 35 ACOs). 

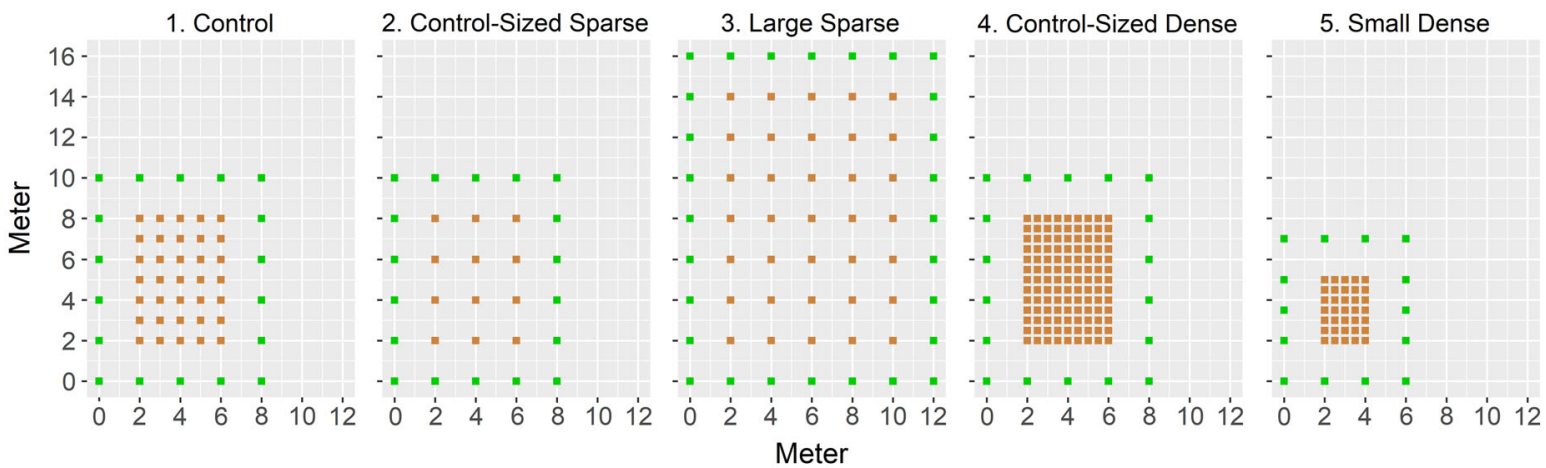

FIG. 2. Five experimental ACO arrays used to survey red-backed salamander populations. From left to right is the control, control-sized sparse, large sparse, control-sized dense, and small dense configurations. ACOs in the experimental arrays are indicated by the brown squares. Also shown are the outer rings of ACOs, which are indicated by the green squares: these are not considered part of the experimental arrays and are therefore not included in our array descriptions. Within sites, arrays were separated by a minimum of $25 \mathrm{~m}$ to avoid captures of individuals at multiple arrays.

It is worth noting that, as a part of another investigation of salamander movement into the arrays from surrounding habitat (see Discussion), each array configuration had an outer ring of ACOs. For each array, the outer ring was placed at the same distance from the array edge and ACOs in all outer rings had identical spacing $(2 \mathrm{~m})$.

ACOs were deployed 14 July 2016 and were left for $67 \mathrm{~d}$, which exceeds the minimum establishment period of one month for ACOs to settle and provide secure refuge and foraging habitat (Otto et al. 2013). Each site was sampled every 10-14 d within red-backed salamander activity windows in Fall 2016 (six occasions), Spring 2017 (six occasions), and Fall 2017 (five occasions). We noted the season (fall or spring) of all captures as activity of red-backed salamanders differs among seasons (Leclair et al. 2008). On each sampling occasion, every ACO was checked for the presence of red-backed salamanders, and individuals occupying ACOs had their exact capture location (ACO ID) recorded and were given unique marks using visual implant elastomer (Phillips and Fries 2009). Temperature has been shown to influence surface presence (i.e., availability) of redbacked salamanders (Feder 1983), and to account for variation in detectability, air temperature was recorded during each visit.

\section{SCR model}

In the context of SCR, point process models describe distributions of organisms in a predetermined state space $(S)$, defined such that it includes the activity center of all individuals exposed to sampling by a given trap array. Our state space was defined as a regular grid of points with $0.25-\mathrm{m}$ resolution within a $3.6-\mathrm{m}$ buffer around the ACOs. The inference objective in SCR is to estimate the number of activity centers (individuals) that exist within the area based on a spatially explicit encounter model.
The encounter model describes the detection data $\left(y_{i j k}\right)$ of each observed individual $(i)$ in each trap $(j)$ on each occasion $(k)$ as Bernoulli random variables $(y=1$ if captured and 0 otherwise)

$$
y_{i j k} \sim \operatorname{Bernoulli}\left(p_{i j k}\right) \text {. }
$$

Specifically, the probability of detecting an individual at a trap $\left(p_{i j k}\right)$ is assumed to decrease with distance between that individual's activity center $\left(\mathbf{s}_{i}\right)$ and the trap location $\left(\mathbf{x}_{j}\right)$. We adopt the commonly applied halfnormal encounter model (although others exist):

$$
p_{i j k}=p_{0 i j k} \times \exp \left(-\operatorname{dist}\left(\mathbf{x}_{j}, \mathbf{s}_{i}\right)^{2} / 2 \sigma^{2}\right)
$$

where $\operatorname{dist}\left(\mathbf{x}_{j}, \mathbf{s}_{i}\right)$ is the distance between a trap and activity center. Here, $\sigma$ is a parameter that, statistically, relates distance to the probability of detections assuming that individuals use areas closer to their activity center more frequently than areas further away, and biologically, is an implied model of space use. Estimation of $\sigma$ is related to the distribution of distances between traps, and our experiment is designed specifically to test for parameter sensitivity to trap configuration.

Spatiotemporal variation in detectability can, in general, be accommodated by modeling $p_{0}$ as a function of occasion-, trap-, and/or individual-specific covariates

$$
\operatorname{logit}\left(p_{i j k}\right)=\beta_{0}+\sum_{v} \beta_{v} X_{v}
$$

where the $\beta_{0}$ is the estimated intercept and $\beta_{1: V}$ are estimated regression coefficients relating detection probabilities to covariates $(X)$ of interest.

We model pixel-specific density, which in this case is assumed to be homogeneous within sessions, using array-level covariates using a log-linear model 


$$
\log \left(D\left(s_{i}\right)\right)=\beta_{0}+\sum_{v} \beta_{v} X_{v} .
$$

Given this model structure, sensitivities of the detection parameters, $\sigma$ and $p_{0}$, to study design may have implications for the estimation of population density. As previously stated, spatial recaptures are paramount in SCR analyses, and failure to generate sufficient spatial recaptures will result in weak estimates. Conventional recommendations for increasing the frequency of spatial recaptures include increasing trapping area (i.e., exposing more individuals to trapping) and increasing trap density (i.e., exposing individuals to more traps; Royle et al. 2013). Variation in density estimates by study design may result from (1) failure to fit an integrated detection function (i.e., the process by which spatial recaptures occur) with data derived from different designs or (2) a behavioral interaction between individuals and trap configuration.

\section{Modeling approach}

It was assumed that populations in the arrays were "closed" to demographic changes within seasons, but birth, death, and dispersal (i.e., immigration, emigration) could occur between seasons, resulting in 45 closed-population "sessions" (15 total arrays, three sampling seasons; see Sutherland et al. 2019 for more on sessions). To account for variation, $p$ was fit with environmental covariates (temperature, season), as well as the effects of site, array, and session, and all possible additive effects (Table 1). Temperature (which the surface activity of red-backed salamanders is sensitive to; Heatwole 1962) and season (in which life history and activity differs; i.e., fall, spring; Petranka 1998) were fit to baseline detection probability on their own and as additive effects with site and design covariates. Preliminary analysis showed consistent and positive behavioral response ("trap happiness"), therefore a behavioral response was specified in all detection models, which accounted for the tendency of individuals to be detected on consecutive sampling occasions (Royle et al. 2013). Additionally, relevant covariates considered for the $\sigma$ parameter included effects of site, array, season, and all possible additive models of the three covariates (Table 1). We observed no spatial recaptures, which are essential for fitting SCR models, in 11 of the 45 sessions, precluding session-specific estimates of $\sigma$. Finally, for the density component of the SCR model, we were primarily interested in variation by array, but also considered effects of site, season, session, and all possible additive effects (Table 1).

Given the large number of covariate effects and additive combinations that could be considered for $p_{0}, \sigma$, and density $(1,296)$, a two-step approach was adopted in which effects on $p$ and $\sigma$ were first considered, with a session-specific density model (i.e., the global model). First, all combinations of $p$ and $\sigma$ model components
TABLE 1. Glossary of covariates used to model each of the three model parameters $\left(p_{0}, \sigma\right.$, density) for evaluating effect of array design on red-backed salamander population parameter estimates.

\begin{tabular}{|c|c|c|}
\hline Covariate & Parameters & Definition \\
\hline- & $\sigma$, density & null model \\
\hline$b$ & $p$ & a trap-specific behavioral response \\
\hline temp & $p$ & $\begin{array}{l}\text { air temperature at time of survey } \\
\text { (used with temp }{ }^{2} \text { to model } \\
\text { quadratic effect) }\end{array}$ \\
\hline temp ${ }^{2}$ & $p$ & $\begin{array}{l}\text { air temperature at time of survey } \\
\text { squared (used with temp to model } \\
\text { quadratic effect) }\end{array}$ \\
\hline Session & $p$, density & $\begin{array}{l}\text { period of demographic closure (i.e., } \\
\text { within site, array, and season) }\end{array}$ \\
\hline Site & $p, \sigma$, density & independent forest study sites \\
\hline Array & $p, \sigma$, density & spatial configuration of ACOs \\
\hline Season & $p, \sigma$, density & sampling window (fall or spring) \\
\hline
\end{tabular}

were fit resulting in a total of 144 models (Appendix S1). Model support was evaluated with the Akaike information criterion (AIC), with a correction for number of individuals due to the small sample size in some sessions. The most supported detection model structure ( $p$ and $\sigma$ ) was then used to investigate variation in density, a total of nine models.

We acknowledge that inference can be sensitive to either trap spacing or behavior, and that these are confounded in the field experiment alone, however, this confounding can be investigated via simulation. We simulated encounter data under each of the five ACO array designs. We used parameter values estimated for the spring season at Site 1 in the control-sized dense array as generating values $(\sigma=1.07 \mathrm{~m}$, density $=$ 1.12 individuals $/ \mathrm{m}^{2}, p_{0}=0.011$ ), as these were the most precise. Site and season were additive effects, so those choices are arbitrary, and we assume the control-sized density estimates are closest to the true values as they are the most precise and fell within estimates of the other array designs, so this choice seemed sensible. To provide more general insight on the effect of array design, we did not simulate temperature effects or a behavioral response on detectability. We generated 100 data sets for each array design, analyzed data using a null SCR model, and compared estimates of $\sigma$ and density among designs (Data S1). We expect that any variation in parameter estimates among array designs in the empirical experiment will also be observed in simulation if it is driven by model sensitivity to array design. All data preparation, processing, and analyses were performed in R (R Core Development Team 2017), and the oSCR package (Sutherland et al. 2019) was used to fit SCR models.

\section{RESULTS}

From 19 September 2016 to 7 November 2017, we made 1,579 captures of 910 individuals among the 15 arrays (Table 2). Using AIC model selection, the first 
TABLE 2. Summary of the average (among sessions) number of captures, individuals, spatial recaptures, and mean maximum distance moved (MMDM) at each of the experimental array designs.

\begin{tabular}{llccc}
\hline \hline & $\begin{array}{c}\text { No. captures } \\
\text { and range }\end{array}$ & $\begin{array}{c}\text { No. } \\
\text { individuals }\end{array}$ & $\begin{array}{c}\text { No. } \\
\text { spatial } \\
\text { captures }\end{array}$ & MMDM \\
\hline Control & $39.56(15-56)$ & 30.56 & 9.56 & 1.51 \\
$\begin{array}{l}\text { Control- } \\
\text { sized sparse }\end{array}$ & $18.33(3-29)$ & 14.44 & 1.67 & 2.78 \\
$\begin{array}{l}\text { Large sparse } \\
\text { Control- }\end{array}$ & $34.56(20-48)$ & 30.56 & 1.56 & 5.05 \\
$\quad$ sized dense & $60.44(41-116)$ & 40.56 & 25.11 & 1.53 \\
Small dense & $17.44(8-25)$ & 14.44 & 3.11 & 1.27 \\
\hline
\end{tabular}

modeling step to identify the best encounter probability model revealed effects of site, array, season, and a quadratic effect of temperature on baseline detection, $p_{0}$ (Appendix S1). It is worth noting that a simpler model without the effect of season was within $2 \mathrm{AIC}_{\mathrm{c}}$ (AIC corrected for sample size) of the top model but, given that the added parameter improved log-likelihood and $\mathrm{AIC}_{\mathrm{c}}$ (despite the added penalty), we chose to include season in the second round of modeling and investigate all potential sources of variation (Arnold 2010). Detection increased with temperature at the time of survey $\left(\beta_{\text {temperature }}=0.054\right.$, SE 0.033$)$ to a peak at $15^{\circ} \mathrm{C}$ before declining again $\left(\beta_{\text {temperature }}^{2}=-0.240\right.$, SE 0.030), and individuals demonstrated a positive trap-specific behavioral response ("trap happy"; $\beta_{\text {behavior }}=1.996, \mathrm{SE}$ $0.140)$. The scale of space use, $\sigma$, varied by season and was larger in spring than in fall $\left(\beta_{\text {spring }}=0.16\right.$, SE 0.06 ; Table 3). An effect of array on $\sigma$ was also supported and was reflective of their respective spatial designs (Fig. 3a; Discussion).

The second modeling step involved investigating variation in density holding the detection model fixed at the structure identified in step 1. The AIC-best model included an additive effect of site, array, and season on density (Table 4; Fig. 3b). Using the control configuration and fall as reference, density was highest in Site 1 (1.72 individuals $/ \mathrm{m}^{2}, 95 \%$ CI $1.21-2.45$ individuals $/ \mathrm{m}^{2}$ ), followed by Site 3 (1.11 individuals $/ \mathrm{m}^{2}, 95 \%$ CI $0.82-$ 1.52 individuals $\left./ \mathrm{m}^{2}\right)$, and lowest in Site 2 (0.94 individuals $/ \mathrm{m}^{2}, 95 \%$ CI $0.68-1.29$ individuals $\left./ \mathrm{m}^{2}\right)$, and was lower in spring than in fall $\left(\beta_{\text {spring }}=-0.326\right.$, SE 0.126; Table 3).

While there was support for the effect of array on density, it is important to note that there was no statistical significance (at the $\alpha=0.05$ level) among arrays except for the control-sized sparse array (Fig. 3b.), i.e., the only array configuration that yielded estimates of density that were different to the control was the control-sized sparse array and was the reason for support for an array effect. This is reflected in the relatively small reduction in the log-likelihood with the addition of the array effect (Table 4).
In our simulation, we found that estimators of density, space use $(\sigma)$, and detection $\left(p_{0}\right)$ were not sensitive to the designs we considered here (Data S1: confirmation simulation.RData). We note that these results are consistent with inference from the experiment about density (see Discussion). However, the simulation results did not mirror the empirical results in relation to $\sigma$, indicating that the variation in $\sigma$ among arrays observed in the field experiment is not simply an artifact of model sensitivity to array design, but an effect of a confounding factor, behavior. Thus, in complementing the empirical results with a simulation, a behavioral response in red-backed salamander space use to array design was revealed.

\section{Discussion}

Given the spatially explicit nature of SCR models, poorly designed trapping arrays have potential to generate biased estimates of density, and while simulation studies have been used to understand design considerations, empirical evaluations of existing design heuristics are limited (Efford and Boulanger 2019). In this novel experiment, we empirically tested the importance of considering model assumptions in designing mark-recapture studies by manipulating the configuration of sampling arrays. With the results of this experiment, we provide an evaluation of the potential for integrating data collected from study designs with different spatial qualities for estimating population densities.

Encouragingly, estimates of density were not sensitive to ACO configuration in our experiment, except for the control-sized sparse design that resulted in the only density estimate that was significantly different than the control. The effect of the control-sized sparse design on density is related to the central role of the model's detection function, which is sensitive to the number of unique individuals encountered and the number of spatial recaptures. Our capture data reveal that the controlsized sparse design resulted in the capture of few unique individuals and few spatial recaptures (Table 2). In this case, and unlike the other designs, few captures and fewer spatial recaptures resulted in estimates of density that were significantly lower than the control. For example, the other sparse design (large sparse array) was larger with more ACOs and therefore exposed more individuals to trapping, providing enough information to achieve reasonable estimates. As can be expected, captures increased with number of traps in the array, which can be seen in the mean captures in the three arrays of the same area but different trap densities (Table 2). Precision was influenced by both the size of the array and the density of the ACOs in the array: the control-sized dense array produced the same estimates of density as the control but with far greater precision (see CIs in Fig. 3b). These results indicate two important features of SCR: first, that estimates of density appear to be robust to variation in spatial sampling because trap configuration is explicitly accounted for, but second, that 
TABle 3. Estimates of density (individuals $\left./ \mathrm{m}^{2}\right), \sigma(\mathrm{m})$, and $p_{0}$, and their $95 \%$ Cis (in parentheses), specific to each categorical covariate found in the AIC-best model from the second round of modeling (site, season, array).

\begin{tabular}{|c|c|c|c|}
\hline Site. season, and array & Density $\left(\right.$ no. $\left./ \mathrm{m}^{2}\right)$ & $p_{0}$ & $\sigma(\mathrm{m})$ \\
\hline \multicolumn{4}{|l|}{1} \\
\hline \multicolumn{4}{|l|}{ Fall } \\
\hline $\mathrm{C}$ & $1.72(1.21-2.45)$ & $0.016(0.011-0.024)$ & $0.95(0.83-1.08)$ \\
\hline $\mathrm{CS}$ & $0.82(0.49-1.37)$ & $0.023(0.013-0.041)$ & $1.01(0.82-1.23)$ \\
\hline LS & $1.31(0.84-2.04)$ & $0.01(0.006-0.016)$ & $1.22(1.09-1.36)$ \\
\hline $\mathrm{CD}$ & $1.56(1.23-1.97)$ & $0.014(0.01-0.018)$ & $0.91(0.82-1.01)$ \\
\hline $\mathrm{SD}$ & $2.08(1.25-3.47)$ & $0.013(0.007-0.023)$ & $0.69(0.53-0.92)$ \\
\hline \multicolumn{4}{|l|}{ Spring } \\
\hline $\mathrm{C}$ & $1.24(0.86-1.81)$ & $0.013(0.009-0.02)$ & $1.11(0.96-1.29)$ \\
\hline $\mathrm{CS}$ & $0.59(0.35-1)$ & $0.019(0.011-0.034)$ & $1.18(0.97-1.45)$ \\
\hline LS & $0.94(0.59-1.5)$ & $0.008(0.005-0.013)$ & $1.43(1.27-1.61)$ \\
\hline $\mathrm{CD}$ & $1.12(0.84-1.5)$ & $0.011(0.008-0.016)$ & $1.07(0.95-1.20)$ \\
\hline $\mathrm{SD}$ & $1.50(0.88-2.56)$ & $0.011(0.006-0.019)$ & $0.81(0.62-1.08)$ \\
\hline \multicolumn{4}{|c|}{ 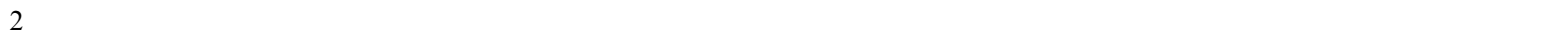 } \\
\hline \multicolumn{4}{|l|}{ Fall } \\
\hline $\mathrm{C}$ & $0.94(0.68-1.29)$ & $0.028(0.019-0.04)$ & $0.95(0.83-1.08)$ \\
\hline $\mathrm{CS}$ & $0.45(0.27-0.72)$ & $0.04(0.024-0.066)$ & $1.01(0.82-1.23)$ \\
\hline LS & $0.71(0.47-1.08)$ & $0.017(0.011-0.026)$ & $1.22(1.09-1.36)$ \\
\hline $\mathrm{CD}$ & $0.85(0.67-1.06)$ & $0.024(0.018-0.031)$ & $0.91(0.82-1.01)$ \\
\hline $\mathrm{SD}$ & $1.13(0.69-1.86)$ & $0.023(0.013-0.039)$ & $0.69(0.53-0.92)$ \\
\hline \multicolumn{4}{|l|}{ Spring } \\
\hline $\mathrm{C}$ & $0.68(0.48-0.95)$ & $0.023(0.015-0.034)$ & $1.11(0.96-1.29)$ \\
\hline $\mathrm{CS}$ & $0.32(0.2-0.53)$ & $0.033(0.019-0.055)$ & $1.18(0.97-1.45)$ \\
\hline LS & $0.51(0.33-0.79)$ & $0.014(0.009-0.022)$ & $1.43(1.27-1.61)$ \\
\hline $\mathrm{CD}$ & $0.61(0.46-0.81)$ & $0.019(0.014-0.027)$ & $1.07(0.95-1.20)$ \\
\hline SD & $0.82(0.49-1.36)$ & $0.019(0.011-0.032)$ & $0.81(0.62-1.08)$ \\
\hline \multicolumn{4}{|c|}{ 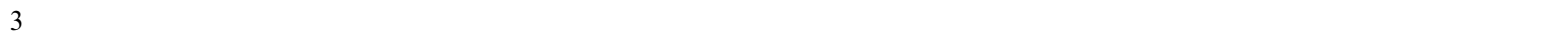 } \\
\hline \multicolumn{4}{|l|}{ Fall } \\
\hline $\mathrm{C}$ & $1.11(0.82-1.52)$ & $0.029(0.02-0.041)$ & $0.95(0.83-1.08)$ \\
\hline $\mathrm{CS}$ & $0.53(0.32-0.86)$ & $0.041(0.024-0.069)$ & $1.01(0.82-1.23)$ \\
\hline LS & $0.85(0.55-1.29)$ & $0.017(0.011-0.027)$ & $1.22(1.09-1.36)$ \\
\hline $\mathrm{CD}$ & $1.00(0.79-1.27)$ & $0.024(0.018-0.033)$ & $0.91(0.82-1.01)$ \\
\hline $\mathrm{SD}$ & $1.34(0.82-2.2)$ & $0.023(0.014-0.04)$ & $0.69(0.53-0.92)$ \\
\hline \multicolumn{4}{|l|}{ Spring } \\
\hline $\mathrm{C}$ & $0.80(0.58-1.11)$ & $0.024(0.016-0.035)$ & $1.11(0.96-1.29)$ \\
\hline $\mathrm{CS}$ & $0.38(0.23-0.63)$ & $0.034(0.02-0.057)$ & $1.18(0.97-1.45)$ \\
\hline LS & $0.61(0.39-0.95)$ & $0.014(0.009-0.023)$ & $1.43(1.27-1.61)$ \\
\hline $\mathrm{CD}$ & $0.73(0.55-0.96)$ & $0.02(0.014-0.028)$ & $1.07(0.95-1.20)$ \\
\hline SD & $0.97(0.58-1.61)$ & $0.019(0.011-0.033)$ & $0.81(0.62-1.08)$ \\
\hline
\end{tabular}

Notes: Baseline detection estimates are reported at the average observed temperature. Additional effects found on baseline detection were a quadratic effect of temperature $\left(\beta_{\text {temperature }}=0.054\right.$, SE $0.033 ; \beta_{\text {temperature }}^{2}=-0.240$, SE 0.030$)$ and a positive behavioral response $\left(\beta_{\text {behavior }}=1.996\right.$, SE 0.140$)$.

the robustness breaks down when there is deviation from general design recommendations. Overall, the similarity in density estimates found in four of the five arrays is a promising result for the future use of SCR for integrating data collection from a variety of spatial sampling designs.

In our simulation study, we find that array design may affect estimates of parameters in the detection function $\left(p_{0}\right.$ and $\sigma$ ) through an effect on behavior. In comparing the results of our simulation to the field experiment, a behavioral effect on space use can be interpreted in that the effect of array on $\sigma$ in our empirical experiment was not observed in simulation, implying that the effect cannot be explained simply by model sensitivity to design. Terrestrial salamanders, particularly plethodontids such as the red-backed salamander, have strict physiological requirements such as moisture-dependent cutaneous respiration. As we previously stated, ACOs are a productive sampling tool because they mimic natural cover (e.g., rocks and logs) that maintain suitable moisture conditions on the soil surface. One explanation for this behavioral interaction is that, because of these physiological constraints, red-backed salamanders do not move freely on the soil surface, but instead move from cover object 

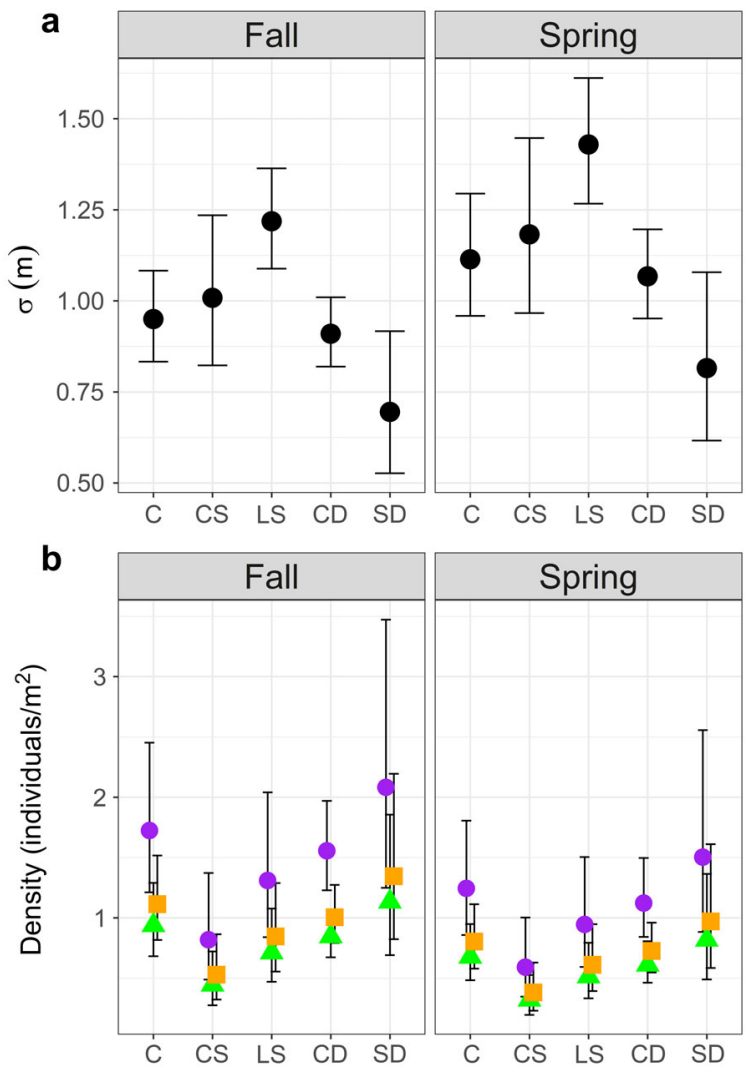

FIG. 3. The (a) $\sigma$ and (b) density estimates produced by the Akaike information criterion (AIC)-best model from the second step of modeling with $95 \%$ CIs. Array is represented on $x$-axes (C, control; CS, control-sized sparse; LS, large sparse; $\mathrm{CD}$, control-sized dense; SD, small dense). In panel $b$, Site 1 estimates are purple circles, Site 2 estimates are green triangles, and Site 3 estimates are orange squares. Fall estimates are depicted in the left panels and spring estimates are in the right panels.

to cover object. Considering this behavior, estimates of $p_{0}$ and $\sigma$ varied intuitively with spatial qualities of the configurations, namely distance between ACOs and the extent of the array (Table 3). Estimates of $\sigma$ were highest in the large sparse array because under this design we were able to detect many individuals and the increased space between ACOs resulted in longer movements (Table 2). Additionally, we could not observe any potential movements that occurred under $2 \mathrm{~m}$ (the minimum distance between ACOs). The small dense design yielded the lowest estimates of $\sigma$, and while it allowed us to observe fine-scale movements, it resulted in encounters of the fewest individuals. These results demonstrate that care should be taken in assigning biological interpretations to estimates of detectability and space use because these are a function of the underlying spatial design and the behavior of the study species.

In the study design process, it is necessary to make assumptions about area and spacing based on the spatial ecology and life history of the study species. Trapping arrays should have a trap spacing capable of generating enough spatial recaptures for accurately estimating $\sigma$, and an area large enough to observe an adequate sample of individuals. We used the best available information on red-backed salamander space use to design the experimental arrays. Previous studies of red-backed salamanders using ACO sampling and SCR analysis estimated $\sigma$ to be 1-3 m (Muñoz et al. 2016b, Sutherland et al. 2016), and therefore, in compliance with SCR study design suggestions (Sollmann et al. 2012), spacing between traps of $2 \mathrm{~m}$ or less should have been sufficient for estimating density. However, baseline detection is appears to vary throughout the range (HernándezPacheco et al. 2019) and with survey conditions (e.g., temperature) making it more difficult to determine the number of traps and spatial extent needed to produce reliable density estimates (Sollmann et al. 2012, Sun et al. 2014, Wilton et al. 2014), explaining the poor performance of our control-sized sparse arrays in estimating density. Variation in baseline detection is particularly consequential for studies like ours in which individuals can only be captured once per survey occasion, as opposed to studies that use passive methods such as camera traps that can detect individuals multiple times within an occasion. This is evidence that, while general recommendations exist, these have mostly been explored in simulation scenarios and assumptions underlying simulation should be considered for each study organism, especially if real conditions differ to that of the simulation scenarios used. Indeed, our simulation of this experiment using parameter estimates from our empirical data failed to detect an effect of array on $\sigma$, implying a behavioral component to this relationship was overlooked by simulation. Clearly, more work is required on the topic of SCR study design and model sensitivity to real system conditions (see Chapter 10 in Royle et al. 2013).

In addition to array design, we found effects of site and season on density. Habitat conditions affecting the physiology of red-backed salamanders vary widely within forests (e.g., moisture, which is necessary for cutaneous respiration; Feder 1983), resulting in variation in abundance even on local scales (Peterman and Semlitsch 2013). Variation in density by season may be explained by annual activity, as courtship and breeding activity take place in the fall on the soil surface (Leclair et al. 2008), perhaps increasing the proportion of surfaceactive salamanders. Further, brooding females do not leave their nesting sites during gestation, which occurs over approximately 6 weeks in the spring (Petranka 1998), likely making them unavailable for capture during this period. While our models did not consider sex, investigation of sex-specific densities may reveal if reproductive behavior is driving seasonal variation. Studies in other parts of the red-backed salamander's range using arrays similar to our control array have found densities that differ from our control estimates of $0.67-1.72$ individuals $/ \mathrm{m}^{2}\left(0.42-0.47\right.$ individuals $/ \mathrm{m}^{2}$ in a Pennsylvania 
TABLE 4. The $\mathrm{AIC}_{\mathrm{c}}$ table produced by the second round of modeling, in which multiple structures on density were evaluated.

\begin{tabular}{|c|c|c|c|c|c|c|c|c|}
\hline Model & $D$ & $p$ & $\sigma$ & $\log L$ & $K$ & $\mathrm{AIC}_{\mathrm{c}}$ & $\Delta \mathrm{AIC}_{\mathrm{c}}$ & $\begin{array}{c}\text { Cumulative } \\
\text { weight }\end{array}$ \\
\hline 1 & $\sim$ site + array + season & $\begin{array}{l}\sim \text { site }+ \text { array }+ \text { season }+ \\
\text { temp }+ \text { temp }^{2}+b\end{array}$ & $\sim$ array + season & 6177 & 25 & 12,406 & 0.0 & 0.56 \\
\hline 2 & $\sim$ site + season & $\begin{array}{l}\sim \text { site }+ \text { array }+ \text { season }+ \\
\text { temp }+ \text { temp }^{2}+b\end{array}$ & $\sim$ array + season & 6182 & 21 & 12,407 & 1.3 & 0.86 \\
\hline 3 & $\sim$ site + array & $\begin{array}{l}\sim \text { site }+ \text { array }+ \text { season }+ \\
\text { temp }+ \text { temp }^{2}+b\end{array}$ & $\sim$ array + season & 6181 & 24 & 12,410 & 4.5 & 0.92 \\
\hline 4 & $\sim$ session & $\begin{array}{l}\sim \text { site }+ \text { array }+ \text { season }+ \\
\text { temp }+ \text { temp }^{2}+b\end{array}$ & $\sim$ array + season & 6140 & 62 & 12,411 & 4.9 & 0.97 \\
\hline 5 & $\sim$ site & $\begin{array}{l}\sim \text { site }+ \text { array }+ \text { season }+ \\
\text { temp }+ \text { temp }^{2}+b\end{array}$ & $\sim$ array + season & 6185 & 20 & 12,412 & 6.0 & 1.00 \\
\hline 6 & $\sim$ array + season & $\begin{array}{l}\sim \text { site }+ \text { array }+ \text { season }+ \\
\text { temp }+ \text { temp }^{2}+b\end{array}$ & $\sim$ array + season & 6187 & 23 & 12,422 & 16.0 & 1.00 \\
\hline 7 & $\sim$ season & $\begin{array}{l}\sim \text { site }+ \text { array }+ \text { season }+ \\
\text { temp }+ \text { temp }^{2}+b\end{array}$ & array + season & 6193 & 19 & 12,425 & 19.0 & 1.00 \\
\hline 8 & $\sim$ array & $\begin{array}{l}\sim \text { site }+ \text { array }+ \text { season }+ \\
\text { temp }+ \text { temp }^{2}+b\end{array}$ & $\sim$ array + season & 6191 & 22 & 12,427 & 21.0 & 1.00 \\
\hline 9 & - & $\begin{array}{l}\sim \text { site }+ \text { array }+ \text { season }+ \\
\text { temp }+ \text { temp }^{2}+b\end{array}$ & $\sim$ array + season & 6197 & 18 & 12,430 & 24.5 & 1.00 \\
\hline
\end{tabular}

Note: $\log L$ is $\log$-likelihood; $K$ is the number of estimated parameters; $\mathrm{AIC}_{\mathrm{c}}$ is the Akaike information criterion corrected for sample size; $\triangle \mathrm{AIC}_{\mathrm{c}}$ is the difference in $\mathrm{AIC}_{\mathrm{c}}$ from the top model; cumulative weight is the sum of the $\mathrm{AIC}_{\mathrm{c}}$ weights of the given and preceding models

population, $1.33-2.16$ individuals $/ \mathrm{m}^{2}$ in a New York population, 2.49-6.26 individuals $/ \mathrm{m}^{2}$ in a Virginia population; Hernández-Pacheco et al. 2019). While it is unclear what the drivers of these differences are, both our study forest and the New York study forest are latitudinally central in the red-backed salamander range, which may explain why our estimates overlap.

A salient criticism of ACOs is that the introduction of artificial habitat elicits immigration to a study area and estimates derived from resulting capture data are ultimately a poor reflection of natural conditions (Siddig et al. 2015). While the simulation revealed evidence of a behavioral interaction between study design and $\sigma$, we note that our inference related to density, our principal interest, is supported by the results of the simulation. In the capture histories, we see that the number of individuals captured decreased with both area and number of ACOs (Table 2), which can be expected in the absence of immigration to an array because area determines the number of activity centers (i.e., individuals) covered and the amount of traps determine the number of opportunities for capture. The effect of attraction across the soil surface on estimates of density may also be understood by evaluating the effect of the outer ring of ACOs (Fig. 2), which were placed to detect movement into or out of arrays. We evaluated the effect of the outer ACOs on our inference of density by removing them (and associated capture data) from the analysis and achieved the same conclusions but with a reduction in precision across all arrays (Appendix S2).

In addition to the attraction of salamanders across the soil surface (i.e., "horizontal" attraction), studies of terrestrial salamanders must also consider a second direction of potential attraction in which fossorial salamanders may be attracted vertically to cover objects on the surface (i.e., increasing the portion of the "superpopulation," which includes surface-active and fossorial individuals, that is present on the surface; Bailey et al. 2004). Subterranean habitat use of red-backed salamanders is not well understood, but experiments suggest individuals may not be active on the surface every fall and spring (Muñoz et al. 2016a), resulting in a varying portion of the population that cannot be detected with surface sampling methods. We observed a "trap-happy" behavioral response in which individuals had an increased probability of detection on consecutive sampling occasions. However, repeat captures of surface-active salamanders may not result from attraction of fossorial individuals to the surface, and resurfacing of active salamanders is also seen in studies that do not use attractive features such as ACOs (Taub 1961). More work is needed to understand surface attraction as a potential mechanism behind the effect of array design on detection parameters.

As we have shown here, a benefit of nonstandardization is that potential study design biases may be revealed by comparing data from multiple designs. However, while this experiment demonstrated that comparison of estimates between study designs may be possible, standardization may still be the preferred approach. The inclusion of array design in the top model is evidence that variation in study design complicates the processes of modeling and interpretation, and indicates that there are risks in assuming study designs are equal in an analysis. Further, added parameters are penalized in AIC model evaluation, and therefore true density models may potentially be masked by models with fewer parameters in corresponding detection components (Arnold 2010). Standardization avoids the need for 
adding parameters that describe variation in study design (Gula and Theuerkauf 2013).

Analyses of population density require knowledge of both distribution and abundance but, without empirical data available for use in rigorous spatial population models (i.e., SCR), these assessments may be limited by biased or imprecise population estimates (Engeman 2003). The ability to incorporate data from various study designs may help resolve data deficiencies but must be done carefully so that accuracy of estimates is not in jeopardy (Link and Sauer 1996). Accommodating common analytical obstacles with the use of robust methods makes integrated data sets increasingly powerful as their scope of inference is widened. SCR methods can account for sources of bias in their parameter estimates, most notably with accommodation of heterogeneity in detection probability (Efford 2004, Royle et al. 2013). Accommodation of sampling biases is made possible by the flexibility of SCR models, and this experiment demonstrated that flexibility by integrating multiple spatial study designs in a single analysis.

Comparison of state variables such as true density among populations is difficult without rich data sets and often precluded by non-standardization. The integration of data derived from different sampling designs allows for more robust analyses that have promise for making stronger conclusions (Tenan et al. 2017, Linden et al. 2018). We stress the importance of systematically distributed range-wide data collection in compensating, as drivers of population demographics and processes can be compared across unique populations within species range (Miller and Grant 2015). While standardization strengthens the inference of monitoring programs, this experiment demonstrates that, where standardized range-wide data are not available, population parameters and their variability may still be estimated where study design varies.

\section{ACKNOWLEDGMENTS}

This research was funded by the National Institute of Food and Agriculture, accession 1009581. Undergraduates from University of Massachusetts Amherst assisted in data collection. This is a contribution of the Salamander Population and Adaptation Network (SPARCnet) and contribution \#767 of the U.S. Geological Survey's Amphibian Research and Monitoring Initiative (ARMI). Any use of trade, product, or firm names is for descriptive purposes only and does not imply endorsement by the United States Government.

\section{Literature Cited}

Arnold, T. W. 2010. Uninformative parameters and model selection using Akaike's Information Criterion. Journal of Wildlife Management 74:1175-1178.

Bailey, L. L., T. R. Simons, and K. H. Pollock. 2004. Comparing population size estimators for plethodontid salamanders. Journal of Herpetology 38:370-380.

Beebee, T. J. C., and R. A. Griffiths. 2005. The amphibian decline crisis: a watershed for conservation biology? Biological Conservation 125:271-285.
Borchers, D. L., and M. G. Efford. 2008. Spatially explicit maximum likelihood methods for capture-recapture studies. Biometrics 64:377-385.

Cabe, P. R., R. B. Page, T. J. Hanlon, M. E. Aldrich, L. Connors, and D. M. Marsh. 2007. Fine-scale population differentiation and gene flow in a terrestrial salamander (Plethodon cinereus) living in continuous habitat. Heredity 98:53-60.

Dupont, G., J. A. Royle, M. A. Nawaz, and C. Sutherland. 2021. Optimal sampling design for spatial capture-recapture. Ecology 102:03262.

Efford, M. G. 2004. Density estimation in live-trapping studies. Oikos 106:598-610.

Efford, M. G., and J. Boulanger. 2019. Fast evaluation of study designs for spatially explicit capture-recapture. Methods in Ecology and Evolution 10:1529-1535.

Engeman, R. M. 2003. More on the need to get the basics right: population indices. Wildlife Society Bulletin 31:286-287.

Feder, M. E. 1983. Integrating the ecology and physiology of plethodontid salamanders. Herpetologica 39:291-310.

Gula, R., and J. Theuerkauf. 2013. The need for standardization in wildlife science: home range estimators as an example. European Journal of Wildlife Research 59:713-718.

Heatwole, H. 1962. Environmental factors influencing local distribution and activity of the salamander, Plethodon cinereus. Ecology 43:460-472.

Hernández-Pacheco, R., C. Sutherland, L. M. Thompson, and K. L. Grayson. 2019. Unexpected spatial population ecology of a widespread terrestrial salamander near its southern range edge. Royal Society Open Science 6:182192.

Hesed, K. M. 2012. Uncovering salamander ecology: a review of coverboard design. Journal of Herpetology 46:442-450.

Leclair, M. H., M. Levasseur, and R. J. Leclair. 2008. Activity and reproductive cycles in the northern populations of the red-backed salamander, Plethodon cinereus. Journal of Herpetology 42:31-38.

Linden, D. W., A. P. K. Sirén, and P. J. Pekins. 2018. Integrating telemetry data into spatial capture-recapture modifies inferences on multi-scale resource selection. Ecosphere 9:e02203.

Link, W. A., and J. R. Sauer. 1996. Extremes in ecology: avoiding the misleading effects of sampling variation in summary analyses. Ecology 77:1633-1640.

Lyons, J. E., M. C. Runge, H. P. Laskowski, and W. L. Kendall. 2010. Monitoring in the context of structured decisionmaking and adaptive management. Journal of Wildlife Management 72:1683-1692.

Miller, D. A. W., and E. H. C. Grant. 2015. Estimating occupancy dynamics for large-scale monitoring networks: Amphibian breeding occupancy across protected areas in the northeast United States. Ecology and Evolution 5:4735-4746.

Muñoz, D. J., K. M. Hesed, H. C. Grant, and D. A. W. Miller. 2016a. Evaluating within-population variability in behavior and demography for the adaptive potential of a dispersallimited species to climate change. Ecology and Evolution 6:8740-8754

Muñoz, D. J., D. A. W. Miller, C. Sutherland, and E. H. C. Grant. 2016b. Using spatial capture-recapture to elucidate population processes and space-use in herpetological studies. Journal of Herpetology 50:570-581.

Nichols, G. E. 1935. The hemlock-white pine-northern hardwood region of Eastern North America. Ecology 16:403-422.

Otis, D. L., K. P. Burnham, G. C. White, and D. R. Anderson. 1978. Statistical inference from capture data on closed animal populations. Wildlife Monographs 62:3-135.

Otto, C. R. V., L. L. Bailey, and G. J. Roloff. 2013. Improving species occupancy estimation when sampling violates the closure assumption. Ecography 36:1299-1309. 
Peterman, W. E., and R. D. Semlitsch. 2013. Fine-scale habitat associations of a terrestrial salamander: the role of environmental gradients and implications for population dynamics. PLoS ONE 8:e62184.

Petranka, J. W. 1998. Salamanders of the United States and Canada. Smithsonian Books, Washington, D.C., USA.

Phillips, C. T., and J. N. Fries. 2009. An evaluation of visible implant elastomer for marking the federally listed fountain darter and the San Marcos salamander. North American Journal of Fisheries Management 29:529-532.

R Core Development Team. 2017. R: a language and environment for statistical computing. R Foundation for Statistical Computing, Vienna, Austria. www.R-project.org

Royle, J. A., R. B. Chandler, R. Sollmann, and B. Gardner. 2013. Spatial capture-recapture. Elsevier, Waltham, Massachusetts, USA.

Royle, J. A., A. K. Fuller, and C. Sutherland. 2018. Unifying population and landscape ecology with spatial capture-recapture. Ecography 41:444-456.

Royle, J. A., and K. V. Young. 2008. A hierarchical model for spatial capture-recapture data. Ecology 89: 2281-2289.

Siddig, A. A. H., A. M. Ellison, and S. Jackson. 2015. Calibrating abundance indices with population size estimators of red back salamanders (Plethodon cinereus) in a New England forest. PeerJ 3:e952.
Sollmann, R., B. Gardner, and J. L. Belant. 2012. How does spatial study design influence density estimates from spatial capture-recapture models? PLoS ONE 7:1-8.

Sun, C. C., A. K. Fuller, and J. A. Royle. 2014. Trap configuration and spacing influence parameter estimates in spatial capture-recapture models. PLoS ONE 9:e88025.

Sutherland, C., D. J. Muñoz, D. A. W. Miller, and E. H. C. Grant. 2016. Spatial capture-recapture: a promising method for analyzing data collected using artificial cover objects. Herpetologica 72:6-12.

Sutherland, C., J. A. Royle, and D. W. Linden. 2019. oSCR: a spatial capture-recapture R package for inference about spatial ecological processes. Ecography 42:1459-1469.

Taub, F. B. 1961. The distribution of the red-backed salamander, Plethodon cinereus, with the soil. Ecology 42:681-698.

Tenan, S., P. Pedrini, N. Bragalanti, C. Groff, and C. Sutherland. 2017. Data integration for inference about spatial processes: a model-based approach to test and account for data inconsistency. PLoS ONE 12:e185588.

Williams, B. K., J. D. Nichols, and M. J. Conroy. 2002. Analysis and Management of Animal Populations. Academic Press, San Diego, California, USA.

Wilton, C. M., E. E. Puckett, J. Beringer, B. Gardner, L. S. Eggert, and J. L. Belant. 2014. Trap array configuration influences estimates and precision of black bear density and abundance. PLoS ONE 9:e111257.

\section{SUPPORTING INFORMATION}

Additional supporting information may be found online at: http://onlinelibrary.wiley.com/doi/10.1002/eap.2419/full 